\title{
EVALUACIÓN DE DOSIS, PERIODOS DE APLICACIÓN Y RESIDUALIDAD DE PACLOBUTRAZOL EN TOMATE
}

\author{
Lucia Juárez-Rodríguez ${ }^{1}$, Mario Pérez-Grajales ${ }^{1}$, Rogelio Castro-Brindis ${ }^{1}$, Antonio Segura- \\ Miranda $^{2}$, Natanael Magaña-Lira ${ }^{1}$ y J. Jesús Magdaleno-Villar ${ }^{1}$
}

RESUMEN

\begin{abstract}
El paclobutrazol (PBZ) es el retardante de crecimiento más utilizado en la manipulación química del crecimiento y desarrollo de plantas hortícolas. Se estudió el efecto de aplicaciones foliares de paclobutrazol en distintas dosis e intervalos de aplicación sobre el crecimiento vegetativo, rendimiento, calidad fisicoquímica y residualidad en frutos de tomate Saladette (Solanum lycopersicum L.), en un ciclo largo de producción en invernadero. El experimento se realizó en 2019 en Chiconcuac, estado de México. Se evaluaron 13 tratamientos de la combinación de tres dosis de PBZ (35, 50 y $\left.65 \mathrm{mg} \cdot \mathrm{L}^{-1}\right)$ y cuatro intervalos de aplicación (30, 40, 50 y 60 días), más un control absoluto. El diseño experimental fue de bloques completos al azar con cuatro repeticiones y seis plantas como unidad experimental. Se cuantificaron variables morfológicas, de rendimiento, calidad fisicoquímica y residuos de PBZ en frutos. Los resultados mostraron que la aplicación de $50 \mathrm{mg} \cdot \mathrm{L}^{-1}$ de $\mathrm{PBZ}$ cada 40 días incrementó significativamente $(P \leq 0,05)$ el rendimiento de frutos por planta; en contraste, disminuyó la altura y el área foliar de la planta, sin afectar el peso seco y firmeza del fruto. Se detectaron residuos de PBZ en la epidermis de frutos que rebasaron los límites máximos de residuos (LMR) permitidos según la Unión Europea y Japón; existió una degradación constante del producto con el tiempo, pero la misma no alcanzó el 50 \% 14 días después de su aplicación. Se encontró que el lavado de frutos con agua simple o detergente eliminó casi la totalidad de los residuos adheridos en la epidermis del fruto y no hubo residuos en el interior.
\end{abstract}

Palabras clave adicionales: Degradación de residuos, PBZ, regulador de crecimiento, Solanum lycopersicum

\begin{abstract}
Evaluation of doses, application periods and residuality of paclobutrazol in tomato

Paclobutrazol (PBZ) is the most widely used growth retardant in the chemical manipulation of the growth and development of horticultural plants. The effect of foliar applications of PBZ in different doses and application intervals on vegetative growth, yield, physicochemical quality and residuality in Saladette tomato fruits (Solanum lycopersicum L.) in a long greenhouse production cycle, was studied. The experiment was carried out in 2019 in Chiconcuac, Mexico State. A total of 13 treatments from the combination of three doses of PBZ (35, 50 and $\left.65 \mathrm{mg} \cdot \mathrm{L}^{-1}\right)$ and four application intervals (30, 40, 50 and 60 days), plus an absolute control, were evaluated. The experiment used a randomized complete block design with four replications and six plants as experimental units. Variables were quantified as morphological, yield, physicochemical quality, and PBZ residues in the fruits. The results showed that applications of $50 \mathrm{mg} \cdot \mathrm{L}^{-1}$ of PBZ every 40 days significantly increased $(P \leq 0.05)$ fruit yield per plant; in contrast, it decreased plant height and leaf area, without affecting dry weight, fresh weight and firmness of fruit. PBZ residues were found in the epidermis of fruits that exceeded the maximum residue limits (MRL) recommended by the European Union and Japan; the degradation of the product over time did not reach $50 \%$ even 14 days after application. Washing with simple water or detergent eliminated almost all residues adhered to the epidermis of the fruit, and there were no residues in the pulp.
\end{abstract}

Additional keywords: Growth regulator, PBZ, residues degradation, Solanum lycopersicum

\section{INTRODUCCIÓN}

La planta de tomate (Solanum lycopersicum L.) presenta hábitos de crecimiento determinado e indeterminado, y en México, la producción comercial de esta especie en invernadero, a menudo, emplea variedades de crecimiento indeterminado. Este sistema de producción se

Recibido: Abril 21, 2021

Aceptado: Octubre 29, 2021

${ }^{1}$ Instituto de Horticultura, Universidad Autónoma Chapingo. e-mail: luciajuarez071094@ gmail.com; perezgm7@yahoo.com.mx; rcbrindis69@gmail.com, mlnatanael@gmail.com, jmagdalenovillar8@gmail.com (autor de correspondencia)

${ }^{2}$ Programa de Protección Vegetal, Universidad Autónoma Chapingo. Chapingo, estado de México, México e-mail: antonioseguramiranda13@gmail.com 
caracteriza por usar densidades de 2 a 3 plantas $\cdot \mathrm{m}^{-2}$. El tallo principal se deja crecer más de $7 \mathrm{~m}$ de longitud para cosechar 15 o más racimos por planta durante un ciclo de producción que puede durar hasta un año (Resh, 2004; Sánchez et al., 2017). Sin embargo, el manejo agronómico de este tipo de plantas se dificulta a medida que se incrementa la longitud del tallo, lo que obliga a los productores a buscar alternativas que les permitan manejar las plantas, incrementar el rendimiento y mejorar la calidad de frutos de tomate.

El paclobutrazol (PBZ) es el retardante de crecimiento más utilizado en la producción de frutales, plantas ornamentales, cereales y hortalizas. Se ha empleado para la manipulación química del crecimiento y desarrollo de las plantas a través de la alteración en los procesos bioquímicos y fisiológicos (Gollagi et al., 2019; Ribeiro et al., 2019), mismos que les brindan a las plantas mayor tolerancia al estrés abiótico, incremento del rendimiento y mejor rasgo fisiológico (Tesfahun, 2018). Los rasgos fisiológicos reportados son mayor contenido de clorofila, modificaciones morfológicas de las hojas (hojas más gruesas, menor área foliar, poros estomáticos más pequeños) y mayor densidad de raíces (Campos et al., 2014; França et al., 2018; Gollagi et al., 2019).

El PBZ es un regulador de crecimiento de la familia de los triazoles que inhibe la biosíntesis de giberelinas al bloquear la acción de monooxigenasas $\mathrm{P} 450$ durante el proceso de conversión de ent-kaureno a ácido ent-kaurenoico (Taiz et al., 2015). Las giberelinas estimulan el alargamiento celular y cuando éstas se inhiben, todavía se produce división celular, pero las nuevas células ya no se alargan. Esto da como resultado plantas con el mismo número de entrenudos, pero comprimidos en una longitud más corta y la producción de brotes con el mismo número de hojas (Gollagi et al., 2019). A pesar de los múltiples beneficios de PBZ Kishore, et al. (2015) señalan que es una molécula ambientalmente estable en suelo y agua, con una vida media de más de un año. En dosis óptimas posee bajo potencial para contaminar aguas subterráneas, pero una vez adherido a los sedimentos el riesgo de exposición a la vida acuática es alta. La toxicidad de PBZ en seres humanos se considera moderadamente peligroso con posible efecto genotóxico y cancerígeno.
Se han realizado diversos estudios sobre la aplicación de PBZ en el cultivo de tomate y los resultados indican que la altura de las plantas se redujo con $50-200 \mathrm{mg} \cdot \mathrm{L}^{-1}$ del producto aplicado por aspersión entre 10 y 15 días después de la siembra (Velázquez et al., 2008; Silva y Faria Junior, 2011; Seleguini et al., 2016). La aplicación foliar de $50 \mathrm{mg} \cdot \mathrm{L}^{-1}$ de $\mathrm{PBZ}$ fue suficiente para obtener plántulas compactas, con mayor materia seca de raíces y sin alterar el área foliar (Seleguini et al., 2013).

En cuanto a su influencia en el rendimiento y calidad de frutos, Ferreira et al. (2017) señalan que la aplicación de $42,5 \mathrm{mg} \cdot \mathrm{L}^{-1}$ de $\mathrm{PBZ}$ en plantaciones de tomate industrial a los 10 días después de la siembra, no alteró los parámetros de producción ni de calidad química y física. De manera similar, Seleguini et al. (2016) encontraron que la aplicación de PBZ por imbibición de semillas y aplicación en plántulas en dosis de 0, 50 y $100 \mathrm{mg} \cdot \mathrm{L}^{-1}$ no afectó la productividad del cultivo, pero, al aumentar las concentraciones de PBZ se incrementó la producción de frutos medianos y pequeños.

Aunque este regulador de crecimiento se ha vuelto común en la producción de tomate, se desconoce su efecto con aplicaciones periódicas sobre la morfología de las plantas, rendimiento y calidad fisicoquímica de frutos. Así mismo, no se han hecho estudios sobre la degradación de residuos en frutos de tomate. Por lo anterior, el objetivo de este trabajo fue evaluar el efecto de aplicaciones foliares de paclobutrazol en distintas dosis e intervalos de aplicación sobre el crecimiento vegetativo, rendimiento, calidad fisicoquímica y residualidad en frutos de tomate, en un ciclo largo de producción bajo invernadero.

\section{MATERIALES Y MÉTODOS}

Ubicación del sitio experimental y material vegetal. El presente trabajo se llevó a cabo en un invernadero de producción comercial ubicado en Chiconcuac, estado de México (19³4'04" N y $\left.98^{\circ} 54^{\prime} 09^{\prime \prime} \mathrm{W}\right)$ durante los meses enero a septiembre de 2019. Se utilizó el híbrido de tomate 'El Cid F1' (Harris Moran, México), el cual presenta hábito de crecimiento indeterminado y fruto tipo Saladette.

Manejo del cultivo. Las semillas se sembraron en bandejas de poliestireno de 200 cavidades en una 
mezcla de sustratos peat moss y vermiculita en proporción 1:1 (v/v). El trasplante se efectuó 37 días después de la siembra (dds) en bolsas de polietileno negro calibre 600 de $50 \times 50 \mathrm{~cm}$, rellenas con sustrato de arena de tezontle (roca volcánica) de partículas menores a $4 \mathrm{~mm}$ de diámetro. La separación entre macetas fue de $60 \mathrm{~cm}$ y 1,6 m entre hileras, se trasplantaron dos plantas por maceta y se obtuvo una densidad de 2,08 plantas $\cdot \mathrm{m}^{-2}$.

El cultivo se desarrolló en condiciones hidropónicas utilizando una solución con la siguiente concentración (en $\mathrm{mg} \cdot \mathrm{L}^{-1}$ ) de nutrimentos minerales (M. Pérez y R. Castro, Univ. Auton. Chapingo. Comunicación personal): N-127, P-77, K-231, Ca-244, Mg-48, S-118, Fe-10, B-1,15, Mn$1, \mathrm{Cu}-0,5$ y $\mathrm{Zn}-0,4$. Las plantas fueron conducidas a un solo tallo, sostenidas del alambre de tutoreo a $4 \mathrm{~m}$ de altura. Cuando las plantas rebasaron $3 \mathrm{~m} \mathrm{de}$ altura, las bases de los tallos fueron acostadas sobre la línea de cultivo. Además, se realizaron otras prácticas culturales como poda de brotes laterales y hojas, polinización con empleo de abejorros (Bombus impatiens $\mathrm{C}$.) y raleo de frutos, así como el control de plagas y enfermedades.

Tratamientos y diseño experimental. Se evaluaron 13 tratamientos producto de la combinación de tres dosis de PBZ $(35,50$ y 65 $\left.\mathrm{mg} \cdot \mathrm{L}^{-1}\right)$ y cuatro intervalos de aplicación $(30,40$, 50 y 60 días), más un control absoluto. El diseño experimental fue de bloques completos al azar con cuatro repeticiones, seis plantas como unidad experimental, de las cuales las dos del centro fueron utilizadas para medir las variables de estudio y evitar que los residuos del producto del tratamiento contiguo llegaran a estas plantas. Asimismo, se colocó una maceta adicional con dos plantas para separar las unidades experimentales (bordura).

Aplicación de PBZ. La aplicación de PBZ se realizó a partir de los 30 dds en la totalidad de plántulas acorde con las dosis correspondientes, excepto en el control absoluto. Las aspersiones posteriores, además de las dosis, consideraron los periodos de aplicación entre los 60 y 210 dds. El número de aplicaciones del PBZ fue de 6, 5, 4 y 3 para los intervalos de 30, 40, 50 y 60 días, respectivamente. Para el tratamiento con mayor dosis y frecuencia de aplicación $\left(65 \mathrm{mg} \cdot \mathrm{L}^{-1}\right.$ de PBZ cada 30 d), se realizó una aspersión adicional para efectuar el análisis de residuos en fruto.

La solución se preparó utilizando la formulación Cultar $25 \mathrm{SC}\left(250 \mathrm{~g} \cdot \mathrm{L}^{-1}\right.$ i.a.) y se diluyó a una concentración específica de acuerdo con las dosis definidas. la aplicación se realizó en las plántulas con un atomizador manual de $1 \mathrm{~L}$ de capacidad, mientras que las aplicaciones posteriores al trasplante se efectuaron con aspersora de mochila manual de 15 L (Firulana, Swissmex, México) y boquillas de cono regulable. En ambos casos las aspersiones fueron dirigidas al follaje hasta el escurrimiento y se aislaron las plantas tratadas con una pantalla plástica para evitar la deriva del PBZ.

Variables cuantificadas. Se midió un total de 14 variables, de las cuales cuatro son de tipo morfológico, cuatro de rendimiento y seis de calidad fisicoquímica de los frutos.

Las variables morfológicas, cuantificadas al final del experimento (219 días después de trasplante (ddt)), fueron la altura de planta medida desde la base del tallo al ápice de crecimiento; diámetro del tallo tomado con un vernier digital a una altura del tallo de $2 \mathrm{~m}$; número de entrenudos; y área foliar determinada mediante un muestreo al azar de dos hojas con desarrollo completo, utilizando un integrador de área foliar LI-3000A.

Las variables de rendimiento, registradas semanalmente, desde el inicio de la cosecha (91 ddt) hasta el corte número 13 (175 ddt) fueron el número total de frutos por planta, correspondiente a la suma de frutos cosechados en cada corte; rendimiento de fruto por planta, peso promedio de fruto; y el número de frutos por racimo (determinado en los primeros seis racimos).

Las variables de calidad de frutos, medidas a los 219 ddt mediante un muestreo al azar de tres frutos por tratamiento y repetición, fueron el peso seco del fruto, luego de secado en estufa a $75^{\circ} \mathrm{C}$ hasta peso constante; grosor de pericarpio medido en la zona ecuatorial del fruto usando un vernier digital; firmeza del fruto determinado en la epidermis y zona ecuatorial del fruto mediante un texturómetro digital Compact Gauge; sólidos solubles totales (SST), cuantificados con un refractómetro digital portátil PAL-1; acidez titulable (AT, como ácido cítrico) según la metodología de AOAC (1990); y la relación SST/AT.

Muestreo de frutos para determinar análisis de residuos. Con la finalidad de obtener la curva de 
degradación de PBZ, se realizó una aplicación adicional del tratamiento con mayor dosis y frecuencia de aplicación $\left(65 \mathrm{mg} \cdot \mathrm{L}^{-1}\right.$ de $\mathrm{PBZ}$ cada 30 días) a los 210 dds. Una hora después de la aplicación, entre 10 y 11 de la mañana se procedió al muestreo de frutos con base en las indicaciones del manual técnico de muestreo de productos agrícolas de la Secretaria de Agricultura Ganadería Desarrollo Rural Pesca y Alimentación (SAGARPA) para determinación de residuos de plaguicidas (SENASICA, 2010). Para ello se consideraron los tratamientos de $65 \mathrm{mg} \cdot \mathrm{L}^{-1}$ de $\mathrm{PBZ}$ cada 30 días y el control absoluto. Para el primero se tomaron cinco muestras, cada una con $1,5 \mathrm{~kg}$ de frutos completos, a los $0,1,3,7$ y 14 días después de la aplicación (dda) con dos repeticiones, mientras que el control absoluto se muestreó el mismo día de la aplicación, con su respectiva repetición. Posteriormente las muestras se congelaron a $-20{ }^{\circ} \mathrm{C}$. Estas fueron extraídas y analizadas mediante la metodología establecida por la AOAC (2007) en el laboratorio CIDAM (Centro de Innovación y Desarrollo Agroalimentario de Michoacán), acreditado por la Entidad Mexicana de Acreditación (EMA).

Durante el periodo de muestreo para el análisis de residuos de PBZ en fruto, las condiciones ambientales registradas en invernadero alcanzaron temperaturas de 32 y $13{ }^{\circ} \mathrm{C}$ en tanto que, la humedad relativa fue de 90 y $35 \%$ (máximas y mínimas, respectivamente).

Lavado de frutos en condiciones de laboratorio. A partir del análisis de residuos de PBZ en frutos de tomate bajo invernadero, se estableció un ensayo adicional en laboratorio con los frutos obtenidos en la etapa final de producción del cultivo (282 ddt), con la finalidad de determinar la presencia de PBZ en la epidermis del fruto de tomate o en el interior de éste.

El muestreo se realizó conforme a lo establecido por SENASICA (2010). En este experimento se evaluaron tres tratamientos: 1 , frutos cosechados del control absoluto y lavados con agua; 2, frutos cosechados del tratamiento con mayor dosis y frecuencia de aplicación $\left(65 \mathrm{mg} \cdot \mathrm{L}^{-1}\right.$ cada $30 \mathrm{~d}$ ) lavados con agua y 3 , frutos del tratamiento con mayor dosis y frecuencia lavados con detergente en polvo al $1 \%$ (sulfato de sodio, dodecilbencensulfonato de sodio, silicato de sodio y carbonato de sodio al 55, 25, 15 y $5 \%$, respectivamente). Los dos últimos tratamientos fueron asperjados con $65 \mathrm{mg} \cdot \mathrm{L}^{-1}$ de $\mathrm{PBZ}$ y se dejaron secar por $24 \mathrm{~h}$, mientras que el control absoluto se asperjó únicamente con agua simple. Los tres tratamientos fueron sometidos a un proceso de lavado durante un minuto y se dejaron secar por un periodo de $5 \mathrm{~h}$.

Se usó un diseño experimental completamente al azar, con dos repeticiones y $0,6 \mathrm{~kg}$ de fruto como unidad experimental. Los frutos fueron congelados y enviados al laboratorio CIDAM para el análisis de residuos, con base a la metodología de la AOAC (2007).

Análisis estadístico. Con los datos obtenidos en el invernadero se realizó un análisis de varianza con el diseño experimental bloques completos al azar. Para variables cuyo efecto fue significativo se ejecutó la comparación de medias de Tukey $(P \leq 0,05)$. El comportamiento de los residuos de PBZ a través del tiempo se ajustó a un modelo de regresión lineal simple y a partir de esta ecuación se estimó el tiempo necesario para una degradación de $50 \%$ de PBZ. Los datos del ensayo de residuos en frutos lavados se analizaron mediante estadística descriptiva. En todos los casos se usó el paquete estadístico SAS v. 9,0 (Cary, NC, USA).

\section{RESULTADOS Y DISCUSIÓN}

El análisis de varianza mostró que los tratamientos presentaron un efecto altamente significativo $(P \leq 0,01)$ y significativo $(P \leq 0,05)$ sobre las variables AL, AF, PSF y REN, SST, respectivamente (Cuadro 1), lo que demuestra que los tratamientos con PBZ afectaron el crecimiento vegetativo y desarrollo reproductivo de las plantas de tomate.

Variables morfológicas. Los tratamientos de PBZ con dosis de $50 \mathrm{mg} \cdot \mathrm{L}^{-1}$ aplicado cada 30 y 40 días, y con dosis de $65 \mathrm{mg} \cdot \mathrm{L}^{-1}$ cada 40 días, redujeron significativamente $(P \leq 0,05)$ la altura de planta con relación al control absoluto a los 219 días después de la siembra (dds) (Cuadro 2). Esta reducción fue de $20 \%(1 \mathrm{~m})$ en promedio, lo que es favorable para el manejo del cultivo. Tesfahun (2018) señala que la disminución de la altura es el resultado morfológico más evidente al aplicar PBZ, esta actividad se asocia con el acortamiento de los entrenudos, en lugar de la reducción del número de entrenudos. 
En el presente estudio se corroboró esta afirmación al no observarse diferencia significativa en el número de entrenudos a los 219 dds, por lo que, la limitación de la altura es consecuencia del modo de acción de PBZ que inhibe la biosíntesis de giberelinas, responsables de la división y elongación celular (Taiz et al., 2015). En otro estudio, Seleguini et al. (2016) encontraron que la altura de las plantas de tomate disminuye el $15 \%$ a los 65 ddt, con relación al control, con la aplicación de $100 \mathrm{mg} \cdot \mathrm{L}^{-1}$ de PBZ a los 15 días después de la siembra.

Cuadro 1. Cuadrados medios del análisis de varianza para las variables evaluadas con distintas dosis y periodos de aplicación de paclobutrazol en tomate

\begin{tabular}{|c|c|c|c|c|c|c|c|c|}
\hline $\begin{array}{l}\text { Fuente de } \\
\text { variación }\end{array}$ & $\begin{array}{l}\text { Grados de } \\
\text { libertad }\end{array}$ & $\begin{array}{l}\text { Altura de } \\
\text { planta (m) }\end{array}$ & $\begin{array}{c}\text { Diámetro } \\
\text { de tallo }(\mathrm{cm})\end{array}$ & $\begin{array}{l}\text { Número de } \\
\text { entrenudos }\end{array}$ & $\begin{array}{c}\text { Área foliar } \\
\left(\mathrm{cm}^{-2}\right)\end{array}$ & $\begin{array}{c}\text { Número } \\
\text { de frutos } \\
\text { por } \\
\text { planta }\end{array}$ & $\begin{array}{l}\text { Rendimiento } \\
\text { de fruto } \\
\left(\mathrm{g} \cdot \text { planta }^{-1}\right)\end{array}$ & $\begin{array}{c}\text { Peso } \\
\text { promedio } \\
\text { de fruto }(\mathrm{g})\end{array}$ \\
\hline Bloque & 3 & $0,64 * *$ & $2,91 *$ & 66,10 & 1918,85 & 6,68 & 0,08 & 17,01 \\
\hline Tratamiento & 12 & $0,33 * *$ & 0,95 & 27,78 & $33534,78 * *$ & 65,48 & $1,80^{*}$ & 101,99 \\
\hline Error & 36 & 0,13 & 0,95 & 27,03 & 2865,60 & 37,18 & 0,78 & 57,35 \\
\hline CV (\%) & & 8,19 & 8,97 & 6,88 & 22,94 & 9,89 & 14,68 & 7,77 \\
\hline $\begin{array}{l}\text { Fuente de } \\
\text { variación }\end{array}$ & $\begin{array}{c}\text { Grados de } \\
\text { libertad }\end{array}$ & $\begin{array}{l}\text { Número de } \\
\text { frutos por } \\
\text { racimo }\end{array}$ & $\begin{array}{l}\text { Peso seco } \\
\text { de fruto }(\mathrm{g})\end{array}$ & $\begin{array}{c}\text { Grosor de } \\
\text { pericarpio } \\
(\mathrm{mm})\end{array}$ & Firmeza $(\mathrm{N})$ & $\begin{array}{c}\text { SST } \\
\left({ }^{\circ} \text { Brix }\right)\end{array}$ & $\begin{array}{c}\text { AT } \\
\text { (Ác. Cítrico) }\end{array}$ & $\begin{array}{l}\text { Relación } \\
\text { SST/AT }\end{array}$ \\
\hline Bloque & 3 & 0,87 & 0,22 & 0,20 & 0,07 & 0,15 & 0,22 & 0,04 \\
\hline Trat & 12 & 0,33 & $1,26 * *$ & 0,67 & 0,62 & $0,28 *$ & 0,23 & 0,07 \\
\hline Error & 36 & 0,45 & 0,24 & 0,34 & 0,37 & 0,11 & 0,35 & 0,07 \\
\hline $\mathrm{CV}(\%)$ & & 10,08 & 13,08 & 9,50 & 26,08 & 9,99 & 19,61 & 23,93 \\
\hline
\end{tabular}

SST: Sólidos solubles totales; AT: Acidez titulable. ** y *: significancia para $P \leq 0,01$ y $P \leq 0,05$, respectivamente

Cuadro 2. Variables morfológicas en plantas de tomate 'El Cid F1' evaluados 219 días después de la siembra en respuesta a las aplicaciones periódicas de paclobutrazol

\begin{tabular}{|c|c|c|c|c|c|}
\hline \multicolumn{2}{|c|}{ Tratamiento } & \multirow{2}{*}{$\begin{array}{l}\text { Altura } \\
(\mathrm{m})\end{array}$} & \multirow{2}{*}{$\begin{array}{l}\text { Diámetro de tallo } \\
(\mathrm{cm})\end{array}$} & \multirow{2}{*}{$\begin{array}{l}\text { Número de } \\
\text { entrenudos }\end{array}$} & \multirow{2}{*}{$\begin{array}{l}\text { Área foliar } \\
\left(\mathrm{cm}^{-2}\right)\end{array}$} \\
\hline Dosis $\left(\mathrm{mg} \cdot \mathrm{L}^{-1}\right)$ & Frecuencia (días) & & & & \\
\hline \multirow{4}{*}{35} & 30 & $4,34 \mathrm{ab}$ & $10,69 \mathrm{a}$ & $73,00 \mathrm{a}$ & $274 \mathrm{~b}$ \\
\hline & 40 & $4,49 \mathrm{ab}$ & $11,05 \mathrm{a}$ & $76,25 \mathrm{a}$ & $237 \mathrm{~b}$ \\
\hline & 50 & $4,40 \mathrm{ab}$ & $10,57 \mathrm{a}$ & $77,75 \mathrm{a}$ & $195 \mathrm{~b}$ \\
\hline & 60 & $4,56 \mathrm{ab}$ & $10,09 \mathrm{a}$ & $73,50 \mathrm{a}$ & $241 \mathrm{~b}$ \\
\hline \multirow{4}{*}{50} & 30 & $4,21 \mathrm{~b}$ & 10,86 a & $74,00 \mathrm{a}$ & $178 \mathrm{~b}$ \\
\hline & 40 & $4,29 \mathrm{~b}$ & $10,39 \mathrm{a}$ & $76,25 \mathrm{a}$ & $178 \mathrm{~b}$ \\
\hline & 50 & $4,40 \mathrm{ab}$ & $11,08 \mathrm{a}$ & $75,00 \mathrm{a}$ & $213 b$ \\
\hline & 60 & $4,70 \mathrm{ab}$ & $11,70 \mathrm{a}$ & $80,00 \mathrm{a}$ & $164 \mathrm{~b}$ \\
\hline \multirow{4}{*}{65} & 30 & $4,46 \mathrm{ab}$ & $11,04 \mathrm{a}$ & $71,00 \mathrm{a}$ & $211 b$ \\
\hline & 40 & $4,06 \mathrm{~b}$ & $10,53 \mathrm{a}$ & $73,25 \mathrm{a}$ & $219 b$ \\
\hline & 50 & $4,70 \mathrm{ab}$ & $10,23 \mathrm{a}$ & $79,75 \mathrm{a}$ & $194 \mathrm{~b}$ \\
\hline & 60 & $4,43 \mathrm{ab}$ & $11,97 \mathrm{a}$ & $76,25 \mathrm{a}$ & $206 \mathrm{~b}$ \\
\hline \multicolumn{2}{|c|}{ Control absoluto } & $5,24 \mathrm{a}$ & $10,84 \mathrm{a}$ & $76,00 \mathrm{a}$ & $521 \mathrm{a}$ \\
\hline \multicolumn{2}{|c|}{ DMSH } & 0,92 & 2,45 & 13,02 & 134,1 \\
\hline
\end{tabular}

DMSH: diferencia mínima significativa honesta. Medias con la misma letra dentro de la columna no difieren estadísticamente según la prueba de Tukey $(P \leq 0,05)$

A los 219 dds, el área foliar disminuyó significativamente $(P \leq 0,05)$ con aplicaciones periódicas de PBZ, respecto a plantas sin tratar. $\mathrm{La}$ disminución fue de $60 \%\left(312 \mathrm{~cm}^{2}\right)$ en promedio; en ese sentido, estudios similares señalan que la reducción drástica del área foliar se compensa con 
el aumento en el grosor de las hojas, masa foliar específica y hojas con un color verde más intenso con lecturas altas de unidades SPAD, que a su vez está directamente relacionado con un mayor contenido de clorofila (Siqueira et al., 2008).

Las plantas tratadas con PBZ forman hojas de color verde oscuro y se caracterizan por presentar mayor contenido de clorofila, tasa fotosintética y longevidad prolongada, lo que permite un proceso fotosintético más eficiente. La abundancia de clorofila en las hojas guarda relación directa con la fotosíntesis, uno de los procesos metabólicos esenciales en las plantas; debido que la clorofila absorbe energía de la luz solar e impulsa así la fijación de carbono (Xia et al., 2018). En ese sentido, Moreno et al. (2021) reportaron reducción del área foliar en 24 y $26 \%$ en plántulas de tomate asperjadas con una o tres aplicaciones de PBZ con dosis de $50 \mathrm{mg} \cdot \mathrm{L}^{-1}$, respectivamente, aunque en el presente estudio la reducción fue superior, probablemente por las dosis empleadas y el mayor número de aplicaciones en algunos tratamientos.

El diámetro de tallo permaneció igual que las plantas no tratadas. Por el contrario, Silva et al. (2014) señalan que el diámetro del tallo de las plántulas de tomate se incrementa con 50 y 100 $\mathrm{mg} \cdot \mathrm{L}^{-1}$ de PBZ (aplicado a los $15 \mathrm{dds}$ ), pero se reduce a concentraciones mayores de $150 \mathrm{mg} \cdot \mathrm{L}^{-1}$.

Parámetros de rendimiento. La comparación de medias mostró incremento significativo $(P \leq 0,05)$ del rendimiento de fruto con aplicaciones de 50 $\mathrm{mg} \cdot \mathrm{L}^{-1}$ de $\mathrm{PBZ}$ cada 40 días, respecto al tratamiento sin aplicar (Cuadro 3). Con esta dosis y periodo, el rendimiento tuvo un incremento de $2,36 \mathrm{~kg}$ por planta (48\%), lo cual puede justificar el costo del producto y de las aplicaciones.

Cuadro 3. Variables de rendimiento en plantas de tomate 'El Cid F1' evaluados a los 91-175 días después de trasplante en respuesta a las aplicaciones periódicas de paclobutrazol

\begin{tabular}{|c|c|c|c|c|c|}
\hline \multicolumn{2}{|c|}{ Tratamiento } & \multirow{2}{*}{$\begin{array}{l}\text { Número de } \\
\text { frutos por planta }\end{array}$} & \multirow{2}{*}{$\begin{array}{l}\text { Rendimiento de } \\
\text { fruto }\left(\mathrm{kg} \cdot \text { planta }^{-1}\right)\end{array}$} & \multirow{2}{*}{$\begin{array}{l}\text { Peso promedio } \\
\text { de fruto }(\mathrm{g})\end{array}$} & \multirow{2}{*}{$\begin{array}{c}\text { Número de } \\
\text { frutos por racimo }\end{array}$} \\
\hline Dosis $\left(\mathrm{mg} \cdot \mathrm{L}^{-1}\right)$ & Frecuencia (días) & & & & \\
\hline \multirow{4}{*}{35} & 30 & $65,00 \mathrm{a}$ & $6,51 \mathrm{ab}$ & 99,98 a & $6,75 \mathrm{a}$ \\
\hline & 40 & $63,37 \mathrm{a}$ & $6,02 \mathrm{ab}$ & 95,04 & $7,50 \mathrm{a}$ \\
\hline & 50 & $63,37 \mathrm{a}$ & $6,43 \mathrm{ab}$ & $101,45 \mathrm{a}$ & $6,50 \mathrm{a}$ \\
\hline & 60 & $62,00 \mathrm{a}$ & $5,99 \mathrm{ab}$ & 96,49 a & $6,75 \mathrm{a}$ \\
\hline \multirow{4}{*}{50} & 30 & $59,50 \mathrm{a}$ & $5,70 \mathrm{ab}$ & 95,63 a & $6,50 \mathrm{a}$ \\
\hline & 40 & $68,50 \mathrm{a}$ & $7,26 \mathrm{a}$ & $105,40 \mathrm{a}$ & $6,50 \mathrm{a}$ \\
\hline & 50 & $64,37 \mathrm{a}$ & $6,60 \mathrm{ab}$ & $102,57 \mathrm{a}$ & $6,50 \mathrm{a}$ \\
\hline & 60 & $63,37 \mathrm{a}$ & $6,12 a b$ & 96,55 a & $7,00 \mathrm{a}$ \\
\hline \multirow{4}{*}{65} & 30 & $62,37 \mathrm{a}$ & $6,39 \mathrm{ab}$ & $102,40 \mathrm{a}$ & $6,75 \mathrm{a}$ \\
\hline & 40 & $62,87 \mathrm{a}$ & $6,03 \mathrm{ab}$ & 95,78 a & $6,50 \mathrm{a}$ \\
\hline & 50 & $54,62 \mathrm{a}$ & $4,82 \mathrm{~b}$ & 88,25 & $6,50 \mathrm{a}$ \\
\hline & 60 & $57,00 \mathrm{a}$ & $5,58 \mathrm{ab}$ & 98,11 & $6,50 \mathrm{a}$ \\
\hline \multicolumn{2}{|c|}{ Control absoluto } & $55,00 \mathrm{a}$ & $4,90 \mathrm{~b}$ & 89,04 a & $6,75 \mathrm{a}$ \\
\hline \multicolumn{2}{|c|}{ DMSH } & 15,27 & 2,21 & 18,97 & 1,69 \\
\hline
\end{tabular}

DMSH: diferencia mínima significativa honesta. Medias con la misma letra dentro de la columna no difieren estadísticamente según la prueba de Tukey $(P \leq 0,05)$

Por su parte, el uso del PBZ no exhibió diferencia estadística $(P>0,05)$ en las variables número de frutos por planta, peso promedio de fruto y número de frutos por racimo. En contraste, Endegena (2019), reportó que la aplicación de paclobutrazol en plantas de tomate (etapa $2-4$ y 6-8 hojas verdaderas) con dosis de 1 a $4 \mathrm{~kg} \cdot \mathrm{ha}^{-1}$ de i.a. incrementó significativamente el rendimiento por planta, el cual se asoció positivamente con el número de frutos por racimo y el número de frutos por planta. Ramos et al. (2021) encontraron que cuatro aplicaciones de $50 \mathrm{mg} \cdot \mathrm{L}^{-1}$ de $\mathrm{PBZ}$ aumentó el número total de frutos y el rendimiento por planta en el híbrido de tomate 'El Cid F1'.

El efecto positivo observado en el rendimiento, posiblemente puede atribuirse a la reducción de la altura y en la limitación del crecimiento vegetativo. De esta forma, se obtienen plantas más compactas y los fotoasimilados dirigidos a la formación de hojas jóvenes se redistribuyen 
hacia el desarrollo de órganos reproductivos, lo que favorece la iniciación floral, número de flores por inflorescencia y cuajado de frutos (Mog et al., 2019), aunque en este caso no hubo diferencias significativas en el número de frutos cosechados.

Parámetros de calidad fisicoquímica de frutos. A pesar de que algunos tratamientos no difirieron estadísticamente del control absoluto, el peso seco de fruto disminuyó entre 0,73 y 2,13 g con relación al control $(5,20 \mathrm{~g})$, siendo los tratamientos con mayores dosis $\left(50\right.$ y $\left.60 \mathrm{mg} \cdot \mathrm{L}^{-1}\right)$ y frecuencia $(30 \mathrm{~d})$ los de mayor influencia (Cuadro 4). Esta disminución del peso seco ante las aplicaciones del PBZ es difícil de explicar y pudiera estar asociada a la tendencia que existió hacia la producción de un menor número de frutos por planta en el tratamiento control (Cuadro 3).

En otras investigaciones, como la de Endegena (2019), se ha reportado un menor peso seco del fruto de tomate ante aplicaciones múltiples del retardante en comparación con una sola aplicación, lo que sugiere que la utilización repetida del producto tiende a afectar esta variable. Resultados semejantes fueron encontrados al utilizar el PBZ en el cultivo del pimiento (Silva et al., 2020).

Cuadro 4. Variables de calidad fisicoquímica de frutos en plantas de tomate 'El Cid $\mathrm{F}_{1}$ ' evaluados a los 219 días después del trasplante en respuesta a las aplicaciones periódicas de paclobutrazol

\begin{tabular}{|c|c|c|c|c|c|c|c|}
\hline \multicolumn{2}{|c|}{ Tratamiento } & \multirow{2}{*}{$\begin{array}{l}\text { Peso seco } \\
\text { del fruto } \\
\text { (g) }\end{array}$} & \multirow{2}{*}{$\begin{array}{l}\text { Grosor del } \\
\text { pericarpio } \\
(\mathrm{mm})\end{array}$} & \multirow{2}{*}{$\begin{array}{l}\text { Firmeza } \\
\text { del fruto } \\
(\mathrm{N})\end{array}$} & \multirow{2}{*}{$\begin{array}{c}\text { Sólidos } \\
\text { solubles totales } \\
\left({ }^{\circ} \text { Brix }\right)\end{array}$} & \multirow{2}{*}{$\begin{array}{c}\text { Acidez } \\
\text { titulable } \\
\text { (\% ac. citr.) }\end{array}$} & \multirow[b]{2}{*}{ SST/AT } \\
\hline $\begin{array}{c}\text { Dosis } \\
\left(\mathrm{mg} \cdot \mathrm{L}^{-1}\right) \\
\end{array}$ & $\begin{array}{c}\text { Frecuencia } \\
\text { (días) }\end{array}$ & & & & & & \\
\hline \multirow{4}{*}{35} & 30 & $4,02 \mathrm{abc}$ & $6,16 \mathrm{a}$ & $2,24 \mathrm{ab}$ & $3,48 \mathrm{a}$ & $3,22 \mathrm{a}$ & $1,08 \mathrm{a}$ \\
\hline & 40 & $4,35 \mathrm{ab}$ & 6,93 a & $2,26 \mathrm{ab}$ & $3,49 \mathrm{a}$ & $2,75 \mathrm{a}$ & $1,37 \mathrm{a}$ \\
\hline & 50 & $3,52 \mathrm{bc}$ & $6,07 \mathrm{a}$ & $2,33 \mathrm{ab}$ & $3,81 \mathrm{a}$ & $2,72 \mathrm{a}$ & $1,45 \mathrm{a}$ \\
\hline & 60 & $3,65 \mathrm{bc}$ & $5,86 \mathrm{a}$ & $2,10 a b$ & $3,83 \mathrm{a}$ & $2,98 \mathrm{a}$ & $1,30 \mathrm{a}$ \\
\hline \multirow{4}{*}{50} & 30 & $3,07 \mathrm{c}$ & $5,93 \mathrm{a}$ & $1,99 \mathrm{ab}$ & $3,14 \mathrm{a}$ & $3,09 \mathrm{a}$ & $1,08 \mathrm{a}$ \\
\hline & 40 & $4,47 \mathrm{ab}$ & $6,31 \mathrm{a}$ & $2,27 \mathrm{ab}$ & $3,09 \mathrm{a}$ & $3,05 \mathrm{a}$ & $1,01 \mathrm{a}$ \\
\hline & 50 & $3,67 \mathrm{bc}$ & $6,45 \mathrm{a}$ & $2,55 \mathrm{ab}$ & $3,11 \mathrm{a}$ & $2,88 \mathrm{a}$ & $1,14 \mathrm{a}$ \\
\hline & 60 & $4,00 \mathrm{abc}$ & $6,20 \mathrm{a}$ & $2,46 a b$ & $3,52 \mathrm{a}$ & $3,14 \mathrm{a}$ & $1,14 \mathrm{a}$ \\
\hline \multirow{4}{*}{65} & 30 & $3,27 \mathrm{bc}$ & $5,96 \mathrm{a}$ & $3,50 \mathrm{a}$ & $3,15 \mathrm{a}$ & $3,13 \mathrm{a}$ & $1,01 \mathrm{a}$ \\
\hline & 40 & $3,45 \mathrm{bc}$ & $5,69 \mathrm{a}$ & $2,02 \mathrm{ab}$ & $3,35 \mathrm{a}$ & $2,76 \mathrm{a}$ & $1,22 \mathrm{a}$ \\
\hline & 50 & $3,40 \mathrm{bc}$ & $6,09 \mathrm{a}$ & $2,39 \mathrm{ab}$ & $3,81 \mathrm{a}$ & $3,45 \mathrm{a}$ & $1,12 \mathrm{a}$ \\
\hline & 60 & $3,55 \mathrm{bc}$ & $6,01 \mathrm{a}$ & $2,22 \mathrm{ab}$ & $3,40 \mathrm{a}$ & $3,03 \mathrm{a}$ & $1,19 \mathrm{a}$ \\
\hline \multicolumn{2}{|c|}{ Control absoluto } & $5,20 \mathrm{a}$ & $7,13 \mathrm{a}$ & $1,97 \mathrm{~b}$ & $3,50 \mathrm{a}$ & $3,46 \mathrm{a}$ & $1,03 \mathrm{a}$ \\
\hline \multicolumn{2}{|c|}{ DMSH } & 1,24 & 1,48 & 1,52 & 0,86 & 1,50 & 0,70 \\
\hline
\end{tabular}

DMSH: diferencia mínima significativa honesta. Medias con la misma letra dentro de la columna no difieren estadísticamente según la prueba de Tukey $(P \leq 0,05)$

En cuanto a firmeza de fruto, se observaron valores mayores en los frutos de plantas tratadas con PBZ, aunque el único tratamiento que superó estadísticamente al control fue el que contenía la dosis de $65 \mathrm{mg} \cdot \mathrm{L}^{-1}$ aplicado cada 30 días, presentando un valor de 3,50 N. Cabe señalar que este tratamiento posee la mayor dosis y frecuencia de aplicación, por lo que se deduce que posiblemente se puede mejorar la firmeza de los frutos combinando estos dos factores. A pesar de ello, todos los tratamientos (incluyendo el control) presentaron valores de firmeza superiores a $1,45 \mathrm{~N}$, valor mínimo reportado para la comercialización de tomate (Batu, 2004). En esta investigación no se encontraron diferencias significativas para grosor de pericarpio, sólidos solubles totales, acidez titulable y relación SST/AT, los cuales presentaron valores medios de $6,21 \mathrm{~mm}, 3,44{ }^{\circ}$ Brix, 3,05\% y 1,6 respectivamente. Ferreira et al. (2017) también indican que la aplicación de $42,5 \mathrm{mg} \cdot \mathrm{L}^{-1}$ de $\mathrm{PBZ}$ en plántulas de tomate a los 10 y 15 dds, no afectó a las variables de calidad físico-química de frutos. Así mismo, Ramos et al. (2021) reportan que la aplicación de $50 \mathrm{mg} \cdot \mathrm{L}^{-1}$ de $\mathrm{PBZ}$ en plántulas de tomate no influyó sobre el contenido de sólidos solubles totales, ni el grosor de pericarpio.

Al final del experimento las aplicaciones continuas de PBZ, independientemente de las dosis 
e intervalos de aplicación, disminuyeron $(P \leq 0,05)$ la altura y el área foliar de las plantas en comparación con el control absoluto. El mejor tratamiento fue 50 $\mathrm{mg} \cdot \mathrm{L}^{-1}$ de PBZ cada 40 días al incrementar significativamente $(P \leq 0,05)$ el rendimiento por planta, disminuir la altura y área foliar; sin afectar peso seco y firmeza de fruto. Cabe señalar que cada tratamiento recibió diferente número de aplicaciones de PBZ; los tratamientos que se aplicaron cada 30,
40, 50 y 60 días recibieron un total de seis, cinco, cuatro $\mathrm{y}$ tres aplicaciones, respectivamente, excepto el tratamiento con $65 \mathrm{mg} \cdot \mathrm{L}^{-1}$ de $\mathrm{PBZ}$ cada 30 d que recibió siete aplicaciones, todos ellos distribuidos a lo largo del ciclo de producción en invernadero.

Degradación de PBZ en frutos de tomate. En la Figura 1 se muestra la degradación del PBZ durante un periodo de 14 días.

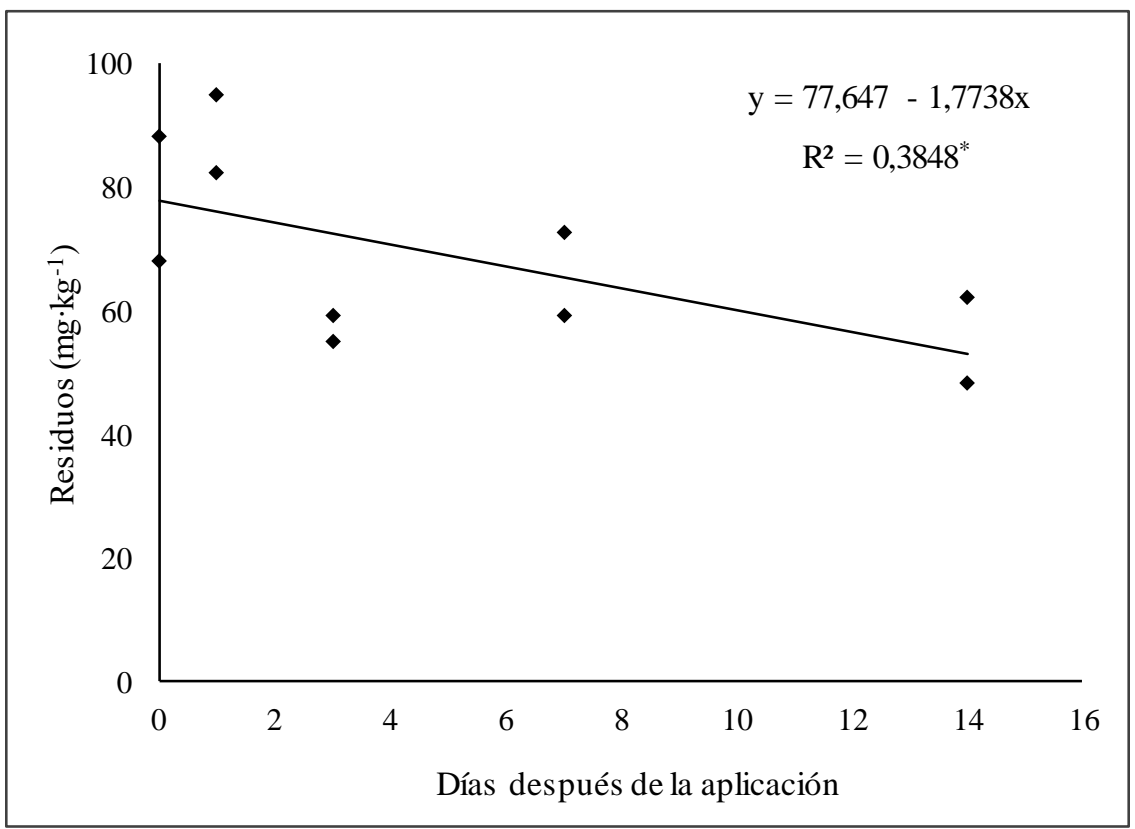

Figura 1. Degradación de residuos de paclobutrazol en frutos de tomate. ${ }^{*} \mathrm{y} * *$ : Significativo para $P \leq 0,05$ y $P \leq 0,01$, respectivamente

Las plantas de tomate tratadas con siete aspersiones de $65 \mathrm{mg} \cdot \mathrm{L}^{-1}$ de $\mathrm{PBZ}$ cada 30 días presentaron frutos con una concentración inicial de $77,64 \mathrm{mg} \cdot \mathrm{kg}^{-1}$. La diferencia entre la concentración asperjada y la concentración inicial de residuos en frutos se debió, probablemente, a un efecto acumulativo de las aplicaciones realizadas. Este mismo comportamiento fue observado por Osuna (2001) y Sharma y Awasthi (2005a) quienes reportan que las aplicaciones continuas de PBZ al suelo en plantaciones de mango incrementaron la cantidad de residuos en el suelo, a la vez que su efecto residual sobre el vigor de los brotes, floración y rendimiento de frutos se extendió por más tiempo. Reddy y Kurian (2008) también reportaron de la cualidad residual de PBZ en el suelo cuando se administró por tres años consecutivos y recomendaron detener la aplicación o reducir la dosis. El efecto residual de una sustancia se debe a su persistencia en el ambiente, que se define como "el tiempo que permanece en un entorno particular antes de que sea transportada físicamente a otro compartimento y/o se transforme química o biológicamente" (Pavan y Worth, 2008). Se ha reportado una alta persistencia de PBZ (210 días) en una capa de suelo de 0 a $15 \mathrm{~cm}$ colectada en distintas zonas de la India (Sharma y Awasthi, 2005b). Así mismo, se ha encontrado persistencia de PBZ en el suelo de hasta por 300 días, después de su aplicación en la base del tallo en el cultivo de mango, mediante el riego (Bhattacherjee y Singh, 2015).

De acuerdo con los resultados obtenidos y los antecedentes reportados, se infiere que el intervalo de aplicación de 30 días no fue suficiente para degradar el PBZ por debajo del LMR permitido para tomate fresco en Japón y la Unión Europea (0,05 y $0,01 \mathrm{mg} \cdot \mathrm{kg}^{-1}$, respectivamente). En este 
sentido, a los 14 d después de la aplicación se encontraron residuos de $52,81 \mathrm{mg} \cdot \mathrm{kg}^{-1}$ que se habían reducido en un $32 \%$ respecto a la concentración inicial (Figura 1). Como se mencionó, este tiempo no fue suficiente para degradar PBZ, lo que refuerza la hipótesis de que la molécula también es persistente en la superficie del fruto de tomate, donde no se tenían reportes.

Con la ecuación de la regresión lineal se estimó que el tiempo de degradación del $50 \%$ de PBZ en frutos de tomate podría estar alrededor de $22 \mathrm{~d}$, por lo que, de continuar la velocidad de degradación observada, la degradación total ocurriría en $44 \mathrm{~d}$. $\mathrm{Al}$ respecto, varios estudios indican que el PBZ aplicado al suelo presentó vida media de 29 a 31 d (Shalini y Sharma, 2006); 41,5 a 43 d (Bhattacherjee y Singh, 2015); y 20 a 64 d (Liu et al., 2015), los cuales son valores similares o superiores a los encontrados en este trabajo.

Lavado de frutos en laboratorio. La forma de lavado de los frutos de tomate mostró resultados variables sobre la cantidad de residuos detectados. En el tratamiento de $65 \mathrm{mg} \cdot \mathrm{L}^{-1}$ de PBZ lavado con detergente se observó menor cantidad de residuos $\left(0,0353 \mathrm{mg} \cdot \mathrm{kg}^{-1}, \sigma=0,0059\right)$ que en el lavado con agua $\left(0,0396 \mathrm{mg} \cdot \mathrm{kg}^{-1}, \sigma=0,0027\right)$. Y como era de esperarse, no se observaron residuos detectables en el tratamiento control. En cualquier caso, el lavado con agua o detergente eliminó casi la totalidad de los residuos de paclobutrazol adheridos en la epidermis del fruto.

Con base en lo anterior, se infiere que con el lavado de frutos con detergente o agua es posible eliminar gran parte de los residuos de PBZ en tomate y se comprueba que éste no se acumula en el interior de los frutos, solo en la epidermis. Al respecto, Berova y Zlatev (2000) descartan la posibilidad de acumulación del retardante en frutos, cuando se aplica en bajas concentraciones, como fue el caso.

Los resultados del análisis de residuos indican que el lavado con agua o detergente en frutos asperjados con la dosis mayor de PBZ $\left(65 \mathrm{mg} \cdot \mathrm{L}^{-1}\right)$ presentaron residuos $\left(\sim 0,03 \mathrm{mg} \cdot \mathrm{kg}^{-1}\right)$ que están por debajo del LMR permitido por las regulaciones de Japón $\left(0,05 \mathrm{mg} \cdot \mathrm{kg}^{-1}\right)$, dado que fueron eliminados de la epidermis del fruto en aproximadamente el $99,95 \%$, lo cual es favorable para el consumo en fresco. Lo anterior concuerda con diversos autores quienes indican que la acumulación de PBZ ocurre mayormente en la superficie de semillas comerciales de pepino (Magnitskiy et al., 2006), cáscara de mandarina (Wang et al., 2014) y epidermis de tubérculos de papa (Liu et al., 2015) y no en el interior. Todos estos autores concuerdan en que el PBZ es capaz de adherirse sólo a la superficie de frutas y hortalizas, por lo que el riesgo en la ingesta de residuos disminuye en productos donde la parte comestible es el mesocarpo (pulpa). No obstante, si se utiliza en productos en los que se consume el exterior del fruto, el riesgo de ingesta de sustancias químicas dañinas para la salud es mayor.

\section{CONCLUSIONES}

La aplicación de $50 \mathrm{mg} \cdot \mathrm{L}^{-1}$ de $\mathrm{PBZ}$ cada 40 días incrementó significativamente $(P \leq 0,05)$ el rendimiento de fruto por planta. En contraste, disminuyó la altura y área foliar sin afectar el peso seco, peso fresco y firmeza de fruto. Luego de las aplicaciones se encontraron residuos elevados en la epidermis del fruto de tomate, que rebasaron los LMR recomendados por regulaciones japonesas $\left(0,05 \mathrm{mg} \cdot \mathrm{kg}^{-1}\right)$ y la Unión Europea $\left(0,01 \mathrm{mg} \cdot \mathrm{kg}^{-1}\right)$.

El tiempo de degradación del $50 \%$ de PBZ adherido en frutos de tomate resultó superior a 14 días en condiciones de invernadero.

En la práctica, todos los residuos de $\mathrm{PBZ}$ adheridos en la epidermis del fruto de tomate, se eliminaron mediante un lavado con agua $o$ detergente.

No se encontraron residuos de PBZ en el interior del fruto de tomate.

\section{AGRADECIMIENTO}

Al Consejo Nacional de Ciencia y Tecnología (CONACYT) por la beca otorgada para la realización de estudios de Maestría en Ciencias en Horticultura, a la Universidad Autónoma Chapingo y al Consejo Particular por su valioso apoyo en la elaboración de este proyecto de investigación.

\section{LITERATURA CITADA}

1. AOAC (Association of Official Analytical Chemists International). 1990. AOAC: Official Methods of Analysis (Volume 1). Arlington, VA, USA. https://n9.cl/7qxw8 (consulta de noviembre 11, 2020).

2. AOAC (Association of Official Analytical 
Chemists International). 2007. AOAC Official Method 2007.01. https://n9.cl/a5t3bp (consulta de noviembre 9, 2020).

3. Batu, A. 2004. Determination of acceptable firmness and color values of tomatoes. Journal of Food Engineering 61(3): 471-475.

4. Berova, M. y Z. Zlatev. 2000. Physiological response and yield of paclobutrazol treated tomato plants (Lycopersicon esculentum Mill.). Plant Growth Regulation 30(2): 117-123.

5. Bhattacherjee, A.K. y V.K. Singh. 2015. Uptake of soil applied paclobutrazol in mango cv. Dashehari and its persistence in soil, leaves and fruits. Indian Journal of Plant Physiology 20(1): 39-43.

6. Campos de Melo, A.P., A. Seleguini, S. Da Rocha y V. Veloso. 2014. Peliculização de sementes de tomate associada ao paclobutrazol. Bragantia 73(2): 123-129.

7. Endegena, W. 2019. Yield and yield-related parameters of tomato (Lycopersicon esculentum Mill.) treated with paclobutrazol at different stages in North West Ethiopia. Journal of Horticulture and Plant Research 6: 37-46.

8. Ferreira, N., E. Vendruscolo, A. Seleguini, W. Dourado, C. Benett y A. Nascimento. 2017. Crescimento, produção e qualidade de frutos de tomateiro em cultivo adensado com uso de paclobutrazol. Revista Colombiana de Ciencias Hortícolas 11(1): 72-79.

9. França, C.F.M., W.S. Ribeiro, M.N.S. Santos, K.P.O.S. Petrucci, E.R. Rêgo y F.L. Finger. 2018. Growth and quality of potted ornamental peppers treated with paclobutrazol. Pesquisa Agropecuária Brasileira 53(3): 316-322.

10.Gollagi, S.G., B.G. Jasmitha y H.S. Sreekanth. 2019. A review on: paclobutrazol a boon for fruit crop production. Journal of Pharmacognosy and Phytochemistry 8(3): 2686-2691.

11.Kishore, K., H.S. Singh y R.M. Kurian. 2015. Paclobutrazol use in perennial fruit crops and its residual effects: a review. Indian Journal of Agricultural Sciences 85(7): 863-872.

12.Liu, H., T. Lin, J. Mao, H. Lu, D. Yang, J. Wang y Q. Li. 2015. Paclobutrazol residue determination in potato and soil using low temperature partition extraction and ultrahigh performance liquid chromatography tandem mass spectrometry. Journal of Analytical Methods in Chemistry 2015: 1-6.

13. Magnitskiy, S., C. Pasian, M. Bennett y J. Metzger. 2006. Effects of soaking cucumber and tomato seeds in paclobutrazol solutions on fruit weight, fruit size, and paclobutrazol level in fruits. Hortscience 41(6): 1446-1448.

14.Mog, B., P. Janani, M.G. Nayak, J.D. Adiga y R. Meena. 2019. Manipulation of vegetative growth and improvement of yield potential of cashew (Anacardium occidentale L.) by Paclobutrazol. Scientia Horticulturae 257: 108748.

15.Moreno-Pérez, E.C., F. Sánchez-del Castillo, M. Ruiz-Díaz y E. Contreras-Magaña. 2021. Effect of population densities and paclobutrazol applications on seedling quality and yield in tomato. Revista Chapingo Serie Horticultura 27(1): 5-17.

16. Osuna-García, J.A. 2001. Residualidad de paclobutrazol en frutos de mango (Mangifera indica L.) 'Tommy Atkins'. Revista Chapingo Serie Horticultura 7(2): 275-282.

17.Pavan, M. y A.P. Worth. 2008. Review of estimation models for biodegradation. QSAR \& Combinatorial Science 27(1): 32-40.

18.Ramos-Fernández, J., O.J. Ayala-Garay, M. Pérez-Grajales, F. Sánchez-del Castillo y J.J. Magdaleno-Villar. 2021. Efecto del paclobutrazol sobre el crecimiento de la planta, rendimiento y calidad del fruto en tomate. Bioagro 33(1): 59-64.

19.Reddy, Y.T.N. y R.M. Kurian. 2008. Cumulative and residual effects of paclobutrazol on growth, yield and fruit quality of 'Alphonso' mango. Journal of Horticultural Sciences 3(2): 119-122.

20.Resh, H. 2004. Cultivos Hidropónicos. 3a ed. Mundi-Prensa. Madrid.

21.Ribeiro, W.S., C.S. Carneiro, C.F.M. Franca, C.M.F. Pinto, P.C.C. Lima, F.L. Finger y F.B. Costa. 2019. Paclobutrazol application in potted ornamental pepper. Horticultura Brasileira 37(4): 464-468.

22.Sánchez-del Castillo, F., E.C. Moreno-Pérez, 
O.A. Pastor-Zarandona y E. Contreras-Magaña. 2017. Disposición de plantas de tomate en doseles en forma de escalera bajo dos densidades de población. Revista Fitotecnia Mexicana 40(3): 333-340.

23. Seleguini, A., E. Pradi-Vendruscolo, L. Cardoso-Campos y M.J. Faria Júnior. 2016. Efeito do paclobutrazol sobre o crescimento de plantas e produzção de tomate (Solanum lycopersicum L.) em ambiente protegido. Scientia Agropecuaria 7(4): 391-399.

24.Seleguini, A., M.J.A. Faria Junior, K.S.S. Benett, O.L. Lemos y S. Seno. 2013. Estratégias para produção de mudas de tomateiro utilizando paclobutrazol. Ciências Agrárias 34(2): 539-548.

25.SENASICA (Servicio Nacional de Sanidad Inocuidad y Calidad Agroalimentaria). 2010. Manual técnico de muestreo de productos agrícolas para determinación de residuos de plaguicidas. México. https://n9.cl/wqa1j (consulta de marzo 11, 2021).

26. Shalini, L. y D. Sharma. 2006. Persistence and movement of paclobutrazol residues in a mango orchard soil. Bulletin of Environmental Contamination and Toxicology 76(6): 930-934.

27.Sharma, D. y M.D. Awasthi. 2005a. Uptake of soil applied paclobutrazol in mango (Mangifera indica L.) and its persistence in fruit and soil. Chemosphere 60(2): 164-169.

28. Sharma, D. y M.D. Awasthi. 2005b. Persistence of paclobutrazol residues in soils of some mango growing areas of India. Pesticide Research Journal 17(1): 82-84.

29.Silva, E.R., M.G. Pinheiro, J.C. Carraro, F.F. Binotti, C.C. Burin, J.N. Oliveira et al. 2020. Cumulative applications of paclobutrazol with leaf nitrogen in Biquinho pepper. Journal of
Experimental Agriculture International 42(5): 64-71.

30.Silva, K.S. y M.J.A. Faria Junior. 2011. Uso de paclobutrazol como estratégia para redução do porte e da brotação lateral de plantas de tomateiro. Ciência e Agrotecnologia 35(3): 539-546.

31.Silva, K.S., M.J.A. Faria Junior, C.G.S. Benett, A. Seleguini y O.L. Lemos. 2014. Utilização de paclobutrazol na produção de mudas de tomateiro. Comunicata Scientiae 5(2): 164-169.

32. Siqueira, D.L., P.R. Cecon y L.C.C. Salomão. 2008. Desenvolvimento do limoeiro "Volkameriano" (Citrus volkameriana Pasq.) submetido a doses de paclobutrazol e ácido giberélico. Revista Brasileira de Fruticultura 30(3): 764-768.

33.Taiz, L., E. Zeiger, I.M. Møller y A. Murphy. 2015. Plant Physiology and Development. Sinauer. Sunderland, MA, USA.

34.Tesfahun, W. 2018. A review on: response of crops to paclobutrazol application. Cogent Food \& Agriculture 4(1): 1-9.

35.Velázquez-Alcaraz, T.J., L. Partida-Ruvalcaba, B. Acosta-Villegas y F. Ayala-Tafoya. 2008. Producción de plantas de tomate y chile aplicando Paclobutrazol al follaje. Universidad y Ciencia 24(1): 21-28.

36.Wang, X., P. Qi, G. Yang, X. Wang, H. Zhang, H. Xu et al. 2014. Enantioselective degradation of (2RS,3RS)-paclobutrazol in peach and mandarin under field conditions. Chirality 26(8): 400-404.

37.Xia, X., Y. Tang, M. Wei y D. Zhao. 2018. Effect of paclobutrazol application on plant photosynthetic performance and leaf greenness of herbaceous peony. Horticulturae 4(1): 5. 
